\title{
Mitteilungen der Österreichischen Gesellschaft für
}

\section{Gynäkologie und Geburtshilfe}

Fortbildungstagung und Jahrestagung der Österreichischen Gesellschaft für Gynäkologie und Geburtshilfe

Bregenz, 1.-4. Juni 1994

Terminplan

Donnerstag, 2. Juni 1994: Fortbildungstagung ganztags;

Freitag, 3. Juni 1994: wissenschaftliche Tagung ganztags;

Samstag, 4. Juni 1994: wissenschaftliche Tagung bis mittags

Kongressort

A-6900 Bregenz Festspiel- und Kongresshaus

Wissenschaftliche A nfragen

Prof. Dr. A. Staudach Landesfrauenklinik Müllner Hauptstrasse 48 A-5020 Salzburg (Österreich)

Tel: (+43/662) 44 82/2501 Fax: (+43/662) 44 82/883

Prof. Dr. H. Salzer

Gynäkologisch-geburtshilflicheAbteilung

Wilhelminenspital

Montlearstrasse 37

A-l 160 Wien (Österreich)

Tel: (+43/1) 49 150/23 44 Fax: (+43/1) 49 150/29 08

Anmeldung und Hotelreservierung

Mondial Congress

Faulmanngasse 4

A-1040 Wien (Österreich)

Tel.: (+43/1) 588040 Fax: (+43/1) 5869185

Kommerzielle und wissenschaftliche Ausstellung

Medizinische Ausstellungs- und Werbegesell-

schaft

M.Rodler\&Co.GmbH

Freyung 6

A-1010 Wien (Österreich)

Tel: (+43/1) 5332935 Fax: (+43/1) 5356016

22 